\title{
Intensive Therapy for Post-resuscitation Disease in a Patient with Hypothermia (Clinical Case)
}

\author{
Alexander F. Potapov ${ }^{1}$, Albina A. Ivanova ${ }^{1, *}$, Alexandra G. Zolotareva ${ }^{2}$, Zakhar M. \\ Rozhin $^{2}$ and Svetlana V. Semenova ${ }^{2}$ \\ ${ }^{1}$ North-Eastern Federal University, Medical Institute, 677000, Yakutsk, Russia \\ ${ }^{2}$ State budgetary institution of the Republic of Sakha (Yakutia) Republic Hospital No. 2 - Center for Emergency \\ Medical Care, 67700 Yakutsk, Russia \\ ${ }^{*}$ Corresponding author. Email: aa.ivanova@s-vfu.ru
}

\begin{abstract}
The present article describes a case of a successful outcome of post-resuscitation disease after prolonged cardiopulmonary resuscitation (CPR) at the prehospital stage in a patient with clinical death due to hypothermia. The unknown, a young man, was admitted to the Emergency Medical Center by an emergency medical team (EMS) after resuscitation measures lasting 240 minutes (4 hours), during which repeated episodes of fibrillation of ventricles and pulse-free electrical activity of the heart were observed. After restoring blood circulation, the patient was hospitalized in the intensive care unit, where a complex of resuscitation measures was continued, which included respiratory and hemodynamic support, prevention of secondary hypoxic damage to the central nervous system and other organs and systems of the body, as well as warming and local treatment of local frostbites. After 72 hours, medicinal sedation was stopped, transmission to spontaneous breathing was carried out in stages, and after 14 days from the moment of hospitalization, the patient in a state of moderate severity, without neurological deficiency and with satisfactory clinical and laboratory indicators was transferred to the department for patients with thermal trauma, where treatment of local frostbites was continued. Thus, a timely and full-fledged set of resuscitation measures at the prehospital stage, adherence to the continuity of treatment of the patient in the hospital made it possible to achieve a favorable outcome in the patient with repeated and prolonged episodes of clinical death.
\end{abstract}

Keywords: post-resuscitation disease, intensive therapy, hypothermia

\section{INTRODUCTION}

Cold trauma remains one of the urgent problems of emergency medicine. As a result of excessive exposure to low temperature, a general (hypothermia, hypothermia) and local (frostbite) cold injury may develop or a combination of them occurs. Its characteristic features are frequent complications from the cardiovascular and respiratory systems in the prereactive and early reactive periods of trauma, as well as necrosis of frozen tissues, leading to amputations of various segments of the limbs and subsequent disability of the patients [1].
Fatal heart rhythm disorders and respiratory disorders as a result of the direct influence of low temperature or reperfusion syndrome, which develops with rapid warming of the patient, are the main causes of death of patients. Therefore, the primary tasks in providing emergency medical care with hypothermia are reduced, both to ensuring adequate oxygenation of the body and maintaining hemodynamics, and to gradually warming the injured person with the observance of the "inside-outside" principle [3]. In cold trauma, there is a high risk of cardiovascular collapse, ventricular fibrillation and thromboembolic complications with careless, abrupt movements of the patient, inefficiency of drugs is noted for hypothermia below $30{ }^{\circ} \mathrm{C}$ and 
possibility of their cumulation and overdose in case of patient warming [3, 4]. All these factors should be taken into account when conducting resuscitation measures for the injured person with hypothermia and frostbites at the prehospital stage, as well as in subsequent intensive care in the hospital.

The European Council for Resuscitation in Death from Hypothermia recommends in the absence of absolute contraindications (the presence of other injuries incompatible with life; impossibility of external heart massage and pulmonary ventilation due to chest glaciation) to perform cardiopulmonary resuscitation (CPR) [5]. This is due to a number of effects of hypothermia - a decrease in the intensity of metabolism and an increase in the resistance of cells and tissues of the body to hypoxia, which have a favorable effect on the course of the post-animation period and a decrease in the severity of neurological disorders [4]. According to one of the latest reviews of management and outcomes in patients with hypothermia and cardiac arrest, the survival rate of this category of patients using extracorporeal support methods (extracorporeal circulation and extracorporeal membrane oxygenation) is approaching $100 \%$. At the same time, the authors of the review cite a number of cases of successful outcomes in patients with hypothermia $17,2{ }^{\circ} \mathrm{C}$ and long-term CPR lasting 8 hours $40 \mathrm{~min}$ [6].

In domestic clinical practice, cases of long-term and successful CPR in hypothermia remain infrequent and in the literature such examples are given extremely rarely. Meanwhile, they are of interest to clinicians to develop a single algorithm for managing this category of patients in the pre-hospital and hospital stages of treatment, developing and implementing domestic intensive care protocols for the early post-animation period.

\section{THE PURPOSE OF THE RESEARCH}

Is to describe the case of a successful outcome of post-resuscitation disease after prolonged CPR at the prehospital stage in a patient with clinical death due to hypothermia.

\section{CASE STUDY}

Unknown, a young man, was admitted to the reception department of the Emergency Medical Center by an emergency medical team (EMS). The patient was found by passers-by on the street unconscious, his time on the street was unknown. According to the official data of the Hydrometeorological Center, the minimum air temperature on the day of the incident (09.02.2021) was $37{ }^{\circ} \mathrm{C}$, humidity $-73-75 \%$, wind speed - 1-2 m/s [2]. The EMS team of anesthesiology-resuscitation arrived at the call site 14 minutes after the call arrived. During the doctor's examination, blood circulation stopped - ventricular fibrillation. In total, the duration of resuscitation measures by the EMS team was 240 minutes (4 hours), during which fibrillation of ventricles and pulse-free electrical activity of the heart (BEAS) were observed. The chronology of resuscitation measures by the EMS team is shown in fig. 1 .

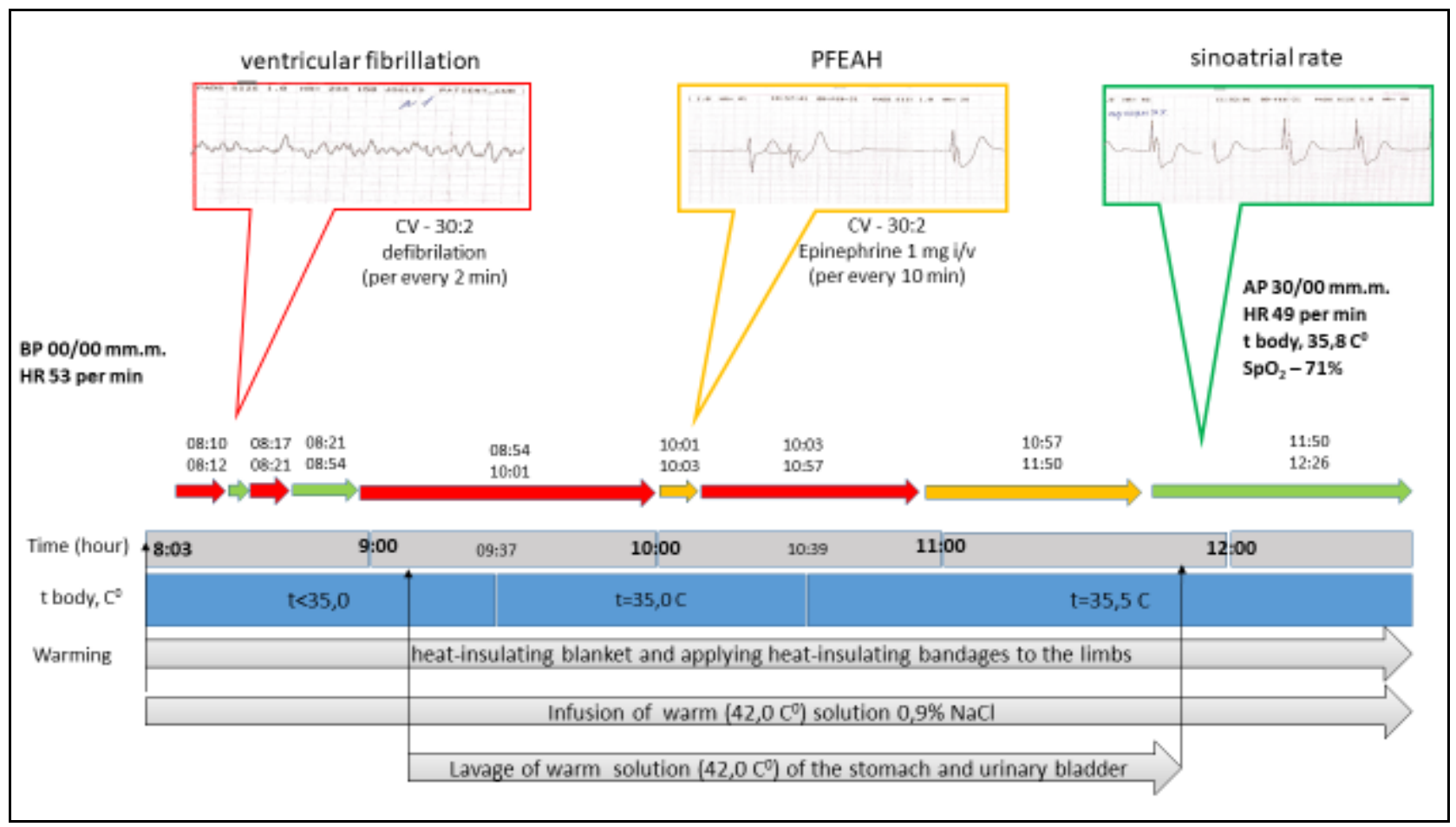

Figure 1. Chronology of cardiopulmonary resuscitation at pre-hospital stage 
After restoration of blood circulation, the patient diagnosed with "General hypothermia. Clinical death, recovery "was hospitalized in the intensive care unit of the Republic Hospital No. 2 - Center for Emergency Medical Care.

Upon admission to the hospital, the patient's condition was extremely serious and was due to acute cardiovascular failure: heart rate (HR) 46 beats per minute, BP 40/20 $\mathrm{mmOg}, \mathrm{SpO} 2$ not defined. On the ECG, atrial fibrillation was determined, the frequency of ventricular contractions was 43 strokes per minute (fig. 2).

Consciousness was absent, coma (6 points by Glasgow scale). There were signs of general hypothermia and local frostbite - frostbite of the lumbar region, heat-insulating bandages were applied on the upper and lower limbs. The internal body temperature of the patient at the prehospital stage and upon admission was not measured. Note that the basis for the diagnosis of Hypothermia, along with the data of history and physical examination, was the measurement of internal body temperature (endaural, esophageal, rectal), which serves as an objective sign of hypothermia of the body and allows assessing its degree.

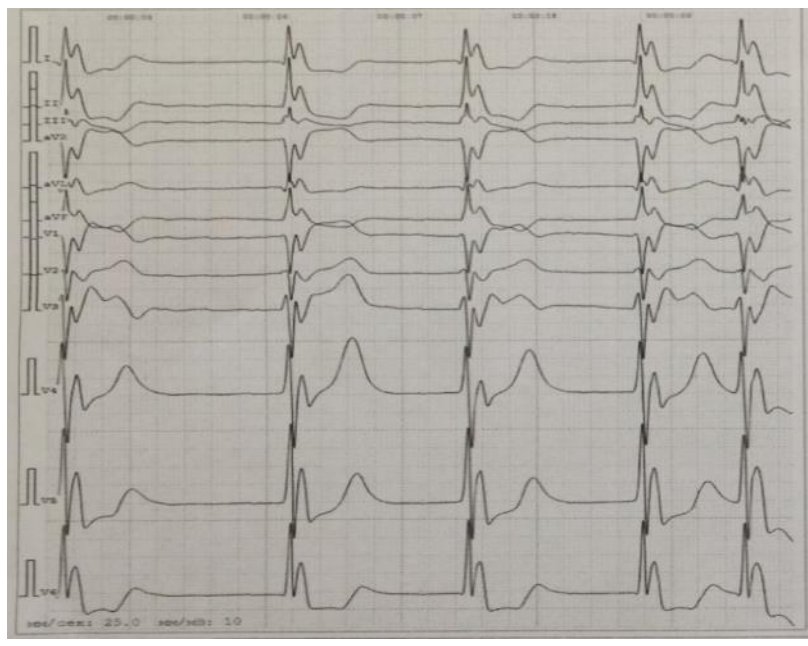

Figure 2. ECG when a patient entered a hospital

In the hospital, the patient was hospitalized in the intensive care unit, where a complex of resuscitation measures was continued, laboratory and instrumental research methods were carried out. The primary task was to maintain hemodynamics. To this purpose, a dopamine microfluid infusion was continued, the initial dose of which was $7 \mu \mathrm{g} / \mathrm{kg} / \mathrm{min}$ with a gradual decrease as hemodynamics stabilized. The basis of infusion therapy was crystalloid solutions, the volume calculation was made taking into account physiological needs and losses to maintain normovolemia. To ensure adequate infusion therapy, catheterization of the right internal jugular vein was performed. Hourly monitoring indicators of hemodynamics in the first 12 hours are shown in fig. 3.

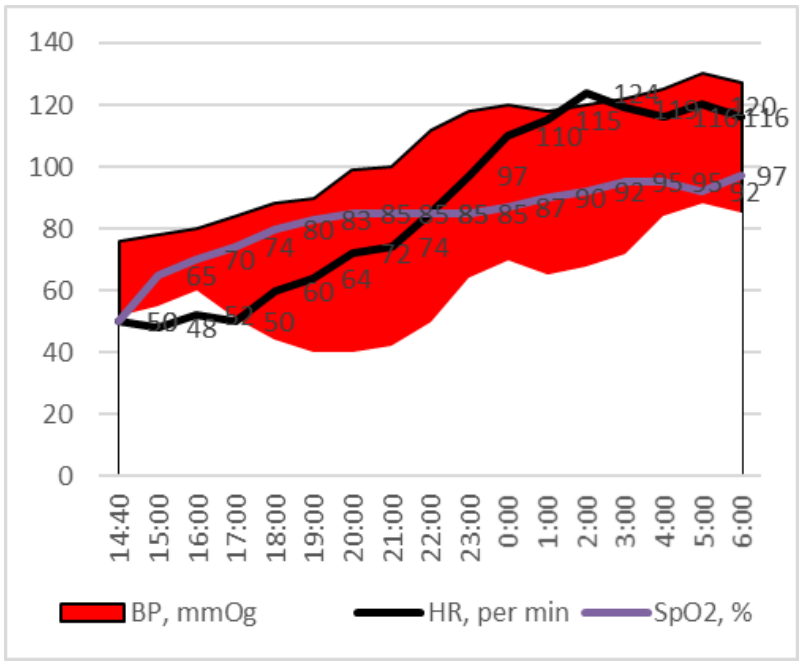

Figure 3. Hourly monitoring rates of hemodynamics in intensive care unit on the first day of hospitalization

Laboratory and instrumental tests were performed at the same time as intensive therapy that revealed pronounced acidosis, increased transaminases, hypoalbuminemia, uremia, hyperglycemia, hypokalemia. The dynamics of the main laboratory indicators in the first 72 hours is presented in Table 1.

The intensive care program, along with hemodynamic support, included active respiratory therapy, prevention of secondary hypoxic damage to the central nervous system and other organs and systems, as well as warming of the patient.

Artificial ventilation of lungs (AVL) was carried out in Pressure control mode with parameters: $\mathrm{P}_{\text {pik }}-20$ см.водн.ст., РЕEP - 7-10 sm water column, $\mathrm{FiO}_{2}-60$ $40 \%$. The target level of respiratory therapy was the maintenance of normoxia ( $\mathrm{pO} 2-37-42 \mathrm{mmHg}$ of venous blood; $\mathrm{SpO} 2 \geq 94 \%$ ) and normocapnia (pCO2 $42-55 \mathrm{mmHg})$.

Due to the repeated and prolonged episodes of clinical death, an important component of intensive care was neurotropic therapy.

Post-resuscitation disease is characterized as multiple organ insufficiency, in which damage to the central nervous system due to anoxia often becomes irreversible and leads to a deep disability of a busy person. The group of neurotropic agents with preferential central action includes a large range of drugs, of which anesthetics are used in the clinical case described by us (in/in the Propofol microfluid $1-1.5 \mathrm{mg} / \mathrm{kg} /$ hour; Sodium oxybutyrate $20-40 \mathrm{mg} / \mathrm{kg} / \mathrm{h}$ ), opioid analgesic (Tramadol $5 \%-2.0, \mathrm{v} / \mathrm{m}$, every 6 hours on the first day), metabolic agent (Cytoflavin, 
$10 \mathrm{ml}, \mathrm{v} / \mathrm{v}$ dropwise in dilution on $0.9 \%-250 \mathrm{ml}$ $\mathrm{NaCl}$ ).

In addition, the patient was given anticoagulant (heparin 20-25 thousand units per day microfluid intravenously under the control of ACTV), antisecretory therapy, antibiotic prophylaxis and nutritional support (probe enteral nutrition with balanced mixtures started in the first 24 hours).

From the moment of admission to the ICU, passive warming of the patient was continued, for this purpose, intravenous infusion of warm crystalloid solutions $\left(42{ }^{\circ} \mathrm{C}\right)$, a heat-insulating blanket and heat-insulating bandages on the limbs were used. Currently, modern high-tech treatments are used for warming, including extracorporeal methods of warming the body, irrigation of body cavities with warmed solutions [3, 5, 6]. Active external warming is considered potentially dangerous, especially when combining hypothermia with frostbite limbs, because it can lead to a paradoxical decrease in internal body temperature due to increased inflow of cold blood from the limbs (afterdrop phenomenon), which increases the likelihood of arterial hypotension and cardiac arrest [6].

On the 4th day of treatment, in order to stabilize the hemodynamics and breathing indices, drug sedation was stopped and after restoring adequate breathing and consciousness, the patient was gradually transferred to spontaneous breathing. The patient in clear consciousness, said hid name, was completely oriented in time and space. At the same time, he didn't remember what happened to him, except drinking the day before. After 14 days, the patient was in a state of moderate severity, in clear consciousness (15 points by Glasgow Coma Scale), with satisfactory clinical and laboratory indicators, was transferred to the department for patients with thermal trauma, where treatment of local frostbites was continued.

Frostbite wounds were preserved in the lumbar, buttocks, left thigh, knee joints and on the feet. In the lumbar region $(A, a)$, and the posterior-lateral surface of the left thigh $(\mathrm{A}, \mathrm{b})$ there were poorly granulating wound, in the knee joint (B) - crusts, on the feet (B) epithelization under the crusts (fig. 4).

The areas of the knee joints were immobilized with longets on the back surface, wounds were treated with antiseptic solutions, bandages with Voskopran wound coatings, bandages with Levomekol ointment and Betadin solution were used.

At the time of preparing the article, the patient was preparing for surgical treatment - autodermoplasty of the wounds of the lumbar region and the hip.
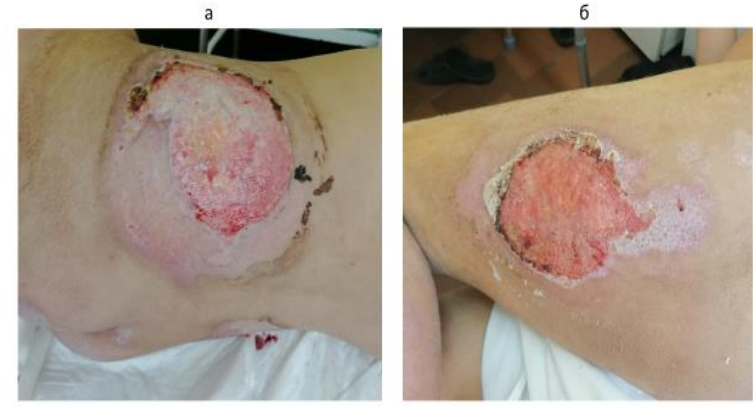

A. Granulating wounds in the lumbar region (a) and on the hip (b)
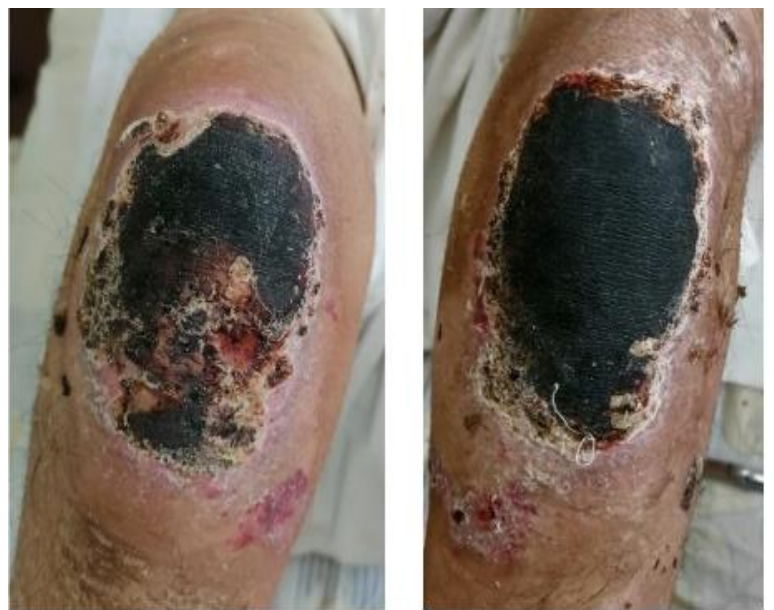

Б. Knee crusts
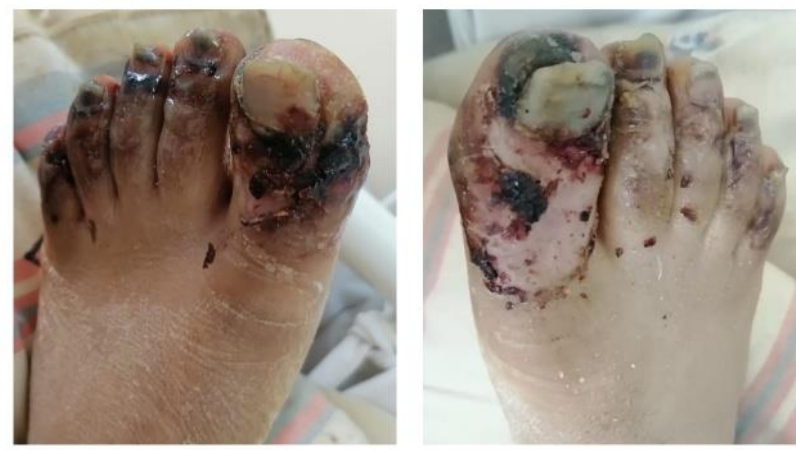

B. Areas of superficial necrosis on toes of feet

Figure 4. Areas of frostbite of the lower extremities (30th day of inpatient treatment).

\section{CONCLUSION}

Thus, despite repeated and prolonged episodes of clinical death, a successful outcome without neurological deficiency was achieved. This was facilitated by both a timely and full-fledged set of resuscitation measures at the prehospital stage and the observance of the continuity of treatment of the patient in the hospital, and the presence of hypothermia, which allows reducing the degree of damage to the central nervous system in critical hypoxia of the body. 


\section{REFERENCES}

[1] Diagnosis and treatment of frostbite (Clinical guidelines), 2017. Retrieved from: http://combustiolog.ru/wpcontent/uploads/2013/07/Diagnostika-i-lechenieotmorozhenij-2017.pdf/

[2] Weather diary (table of daily averages Weather in Yakutsk in February 2021). Retrieved from: https://weatherarchive.ru/Temperature/Yakutsk/Fe bruary-2021/

[3] Cold injuries guidelines, State Alaska, 2014. Retrieved from: http://dhss.alaska.gov/dph /emergency/documents /ems/documents/alaska/

[4] B.M. Monika, D. Martin, E.L. Stefan et fl., The Bernese Hypothermia Algorithm: A consensus paper on in-hospital decision-making and treatment of patients in hypothermic cardiac arrest at an alpine level 1 trauma centre, Injury 42(5) (2011) 539-543. Retrieved from: https://doi.org/10.1016/j.injury.2010.11.037

[5] Europen Resuscitation Council Guidelines for Resuscitation, 2015. Retrieved from: https://www.resuscitationjournal.com/article/S0300 -9572(15)00343-3/fulltext

[6] P. Paal, G. Strapazzon, M.B. Maeder et al., Accidental hypothermia-an update The content of this review is endorsed by the International Commission for Mountain Emergency Medicine (ICAR MEDCOM), Scand. J. of Trauma, Resuscitat. and Emerg. Med. 24 (2016) 111. DOI 10.1186/s13049-016-0303-7 